ARTICLE

\title{
High-fidelity single-shot readout of single electron spin in diamond with spin-to-charge conversion
}

\author{
Qi Zhang (1) 1,2,3,4, Yuhang Guo (1) 1,2,3,4, Wentao Ji (1) 1,2,3,4, Mengqi Wang ${ }^{1,2,3}$, Jun Yin ${ }^{1,2,3}$, Fei Kong (1) 1,2,3, \\ Yiheng Lin ${ }^{1,2,3}$, Chunming Yin (1) ${ }^{1,2,3}$, Fazhan Shi (i) ${ }^{1,2,3}$, Ya Wang (i) ${ }^{1,2,3 凶}$ \& Jiangfeng Du (1) 1,2,3凶
}

High fidelity single-shot readout of qubits is a crucial component for fault-tolerant quantum computing and scalable quantum networks. In recent years, the nitrogen-vacancy (NV) center in diamond has risen as a leading platform for the above applications. The current single-shot readout of the NV electron spin relies on resonance fluorescence method at cryogenic temperature. However, the spin-flip process interrupts the optical cycling transition, therefore, limits the readout fidelity. Here, we introduce a spin-to-charge conversion method assisted by near-infrared (NIR) light to suppress the spin-flip error. This method leverages high spin-selectivity of cryogenic resonance excitation and flexibility of photoionization. We achieve an overall fidelity $>95 \%$ for the single-shot readout of an NV center electron spin in the presence of high strain and fast spin-flip process. With further improvements, this technique has the potential to achieve spin readout fidelity exceeding the fault-tolerant threshold, and may also find applications on integrated optoelectronic devices.

\footnotetext{
${ }^{1}$ Hefei National Laboratory for Physical Sciences at the Microscale and Department of Modern Physics, University of Science and Technology of China, Hefei, China. ${ }^{2}$ CAS Key Laboratory of Microscale Magnetic Resonance, University of Science and Technology of China, Hefei, China. ${ }^{3}$ Synergetic Innovation Center of Quantum Information and Quantum Physics, University of Science and Technology of China, Hefei, China. ${ }^{4}$ These authors contributed equally: Qi

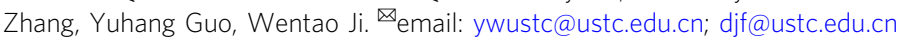


R esonance fluorescence method has become a commonly used method to achieve the single-shot readout of various solid-state spins such as quantum $\operatorname{dot}^{1,2}$, rare-earth ions in crystals $^{3,4}$, silicon-vacancy center ${ }^{5,6}$, and nitrogen-vacancy (NV) center $^{7}$ in diamond. Under spin-selective excitation of optical cycling transition, the spin state is inferred according to collected spin-dependent fluorescence photon counts. However, the accompanying spin non-conservation processes usually limit the optical readout window for photon collection and induce the spin state flip error. This effect has become a significant obstacle for achieving high-fidelity single-shot readout, in particular, to exceed the fault-tolerant threshold ${ }^{8-12}$.

A powerful method to suppress this effect is to explore optical structures for the emitters. The microstructure, such as a solidstate immersion lens, is widely used to enhance the fluorescence collection efficiency ${ }^{7,13-16}$. High-quality nano-cavities strongly coupled to these quantum emitters could even enhance the photon emission rate by orders of magnitude ${ }^{3-6}$. Despite these significant achievements, the practical application of such a highquality cavity remains technically challenging. Extensive engineering works are required to obtain the high-quality cavity, place the emitter into the optimal cavity position, and tune the frequency on-demand. Besides, the fabrication process introduces unwanted strain and surface defects ${ }^{17}$, which may degrade the spin and optical properties ${ }^{7}$.

Here, we demonstrate a new method to achieve a single-shot readout of $\mathrm{NV}$ center electron spin by combing a spin-selective photoionization process. The spin state is on-demand converted into charge state before the spin-flip relaxation becomes significant (Fig. 1a, b). Then the charge state is measured with near unity fidelity thanks to their stability under optical illumination. The essence of this approach is to enhance the ratio of ionization rate $\left(\Gamma_{i o n}\right)$ to the spin-flip rate $\left(\Gamma_{\text {flip }}\right)$.

\section{Results}

The experiments are performed on a bulk NV center inside a solid immersion lens at a cryogenic temperature of $8 \mathrm{~K}$. The measurement scheme utilizes the cycling transition $E_{\mathrm{y}}$ that connects excited and ground states with spin projection $m_{\mathrm{S}}=0$ (Fig. 1a), and the $E_{1,2}$ transition connecting states with spin projection $m_{\mathrm{S}}= \pm 1$. The corresponding optical transitions is shown in Fig. 1c. The fabrication of the solid immersion lens introduced non-axial strain $\delta=5.9 \mathrm{GHz}$ to the NV center used. Therefore, a spin-flip rate $\Gamma_{\text {flip }}$ of $0.75 \pm 0.02 \mathrm{MHz}$ is observed (Fig. 1d), much faster than previously reported $0.2 \mathrm{MHz}$ with low strains ${ }^{7}$. Under selective excitation of $E_{\mathrm{y}}$, spin state $|0\rangle$ could be pumped to the excited state, and be further ionized to charge state $\mathrm{NV}^{0}$ under another NIR laser excitation (1064 nm, Fig. 1a). In contrast, $| \pm 1\rangle$ will not be excited and stay at charge state $\mathrm{NV}^{-}$. Such a deterministic SCC differs from previous work using non-resonant excitation to enhance the readout efficiency of NV center ${ }^{18-23}$.

To verify the photoionization process, we first characterize the charge state readout. Under simultaneous excitation of $E_{\mathrm{y}}$ and $E_{1,2}$ transitions, $\mathrm{NV}^{-}$emits photons regardless of the spin state, while leaving $\mathrm{NV}^{0}$ in the unexcited dark state. The charge state can thus be determined from the detected photon number during the integration window. We evaluate the charge readout fidelity by measuring the correlation between two consecutive readouts (Fig. 2a). The correlation results with an integration window of $500 \mu \mathrm{s}$ is shown in Fig. $2 \mathrm{~b}$ and the statistical distribution of the photon number is shown in Fig. 2c. As expected, the $\mathrm{NV}^{-}$state is distinguishable from the $\mathrm{NV}^{0}$ state according to the photon counts (Fig. 2c). More importantly, a strong positive correlation is observed, except for six anti-correlation cases. And all these anticorrelation cases (circles in Fig. 2b) come from initial $\mathrm{NV}^{-}$ transforming to $\mathrm{NV}^{0}$. This indicates a unity readout fidelity for $\mathrm{NV}^{0}$ state and $99.92 \pm 0.03 \%$ readout fidelity for $\mathrm{NV}^{-}$state. To

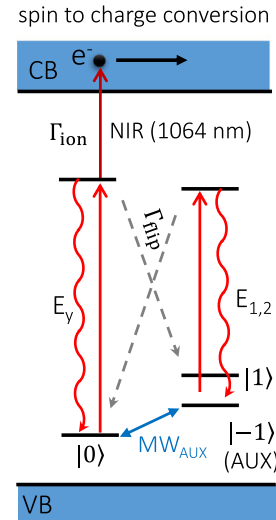

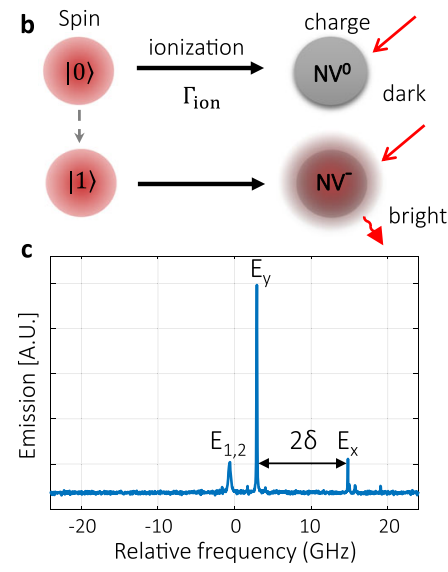

Fig. 1 Single-shot readout scheme based on SCC. a Energy levels used to achieve SCC. Qubit is encoded in the ground state $|0\rangle$ and $|1\rangle$, and the $|-1\rangle=|A \cup X\rangle$ state acts as the auxiliary level. The magnetic field of $585 \mathrm{G}$ aligned to NV axis lifts the degeneracy between $|-1\rangle$ and $|+1\rangle$. Note that the magnitude and direction of the magnetic field used here is not special. The coherent manipulation between $|0\rangle$ and $| \pm 1\rangle$ can be realized by resonant microwave, labeled by blue arrows. $E_{\mathrm{y}}\left(E_{1,2}\right)$ corresponds to the optical transition of the $m_{\mathrm{S}}=0\left(m_{\mathrm{S}}= \pm 1\right)$ state. The counts rate is proportional to the excited state emission rate and the fluorescence photon collection efficiency. The key part of SCC is to ionize (dark red arrow) the excited states of $m_{\mathrm{S}}=0$ before it substantially relaxes to the ground $| \pm 1\rangle$ states through the spin-flip relaxation process (gray dashed arrow). CB is the conduction band of diamond and $V B$ the valance band. $\Gamma_{\text {ion }}$ denotes the ionize rate, and $\Gamma_{\text {flip }}$ denotes the spin-flip rate from the $E_{\mathrm{y}}$ excited state to the ground $| \pm 1\rangle$ states. $A$ more detailed model is in Supplementary information (SI). b A schematic diagram of SCC readout. Under the illumination of $637 \mathrm{~nm}$ laser, NV- keeps fluorescing stably for a long time, while $\mathrm{NV}^{0}$ is not excited. $\mathbf{c}$ The excitation spectrum of the NV center used here at cryogenic temperature of $8 \mathrm{~K}$. Frequency is given relative to $470.4675 \mathrm{THz}(637.2225 \mathrm{~nm})$. The non-axial strain $(\delta)$ induces a splitting of $2 \delta=11.8 \mathrm{GHz}$ between $E_{\mathrm{y}}$ and $E_{\mathrm{x}}$ transitions ${ }^{45}$. d Spin-flip process induces the photoluminescence $(\mathrm{PL})$ decay under $E_{\mathrm{y}}$ excitation $(5.7 \mathrm{nW}$, saturation power $\sim 13 \mathrm{nW})$ with NV initially prepared in $|0\rangle$. At the final equilibrium of $\mathrm{PL}$ decay curve, the NV spin is pumped into $| \pm 1\rangle$. The solid line is the simulation according to the model described in $\mathrm{Sl}$, with the best-fitted spin-flip rate $\Gamma_{\text {flip }}=$ $0.75 \pm 0.02 \mathrm{MHz}$. Inset: PL decay for NV initialized to $| \pm 1\rangle$ under $E_{1,2}$ excitation ( $4.2 \mathrm{nW}$, saturation power $34 \mathrm{nW}$ ). From the PL decay curves, the spin initialization fidelity is estimated to be $99.7 \pm 0.1 \%$ for $| \pm 1\rangle$ subspace and $99.8 \pm 0.1 \%$ for $|0\rangle(\mathrm{SI})$. 

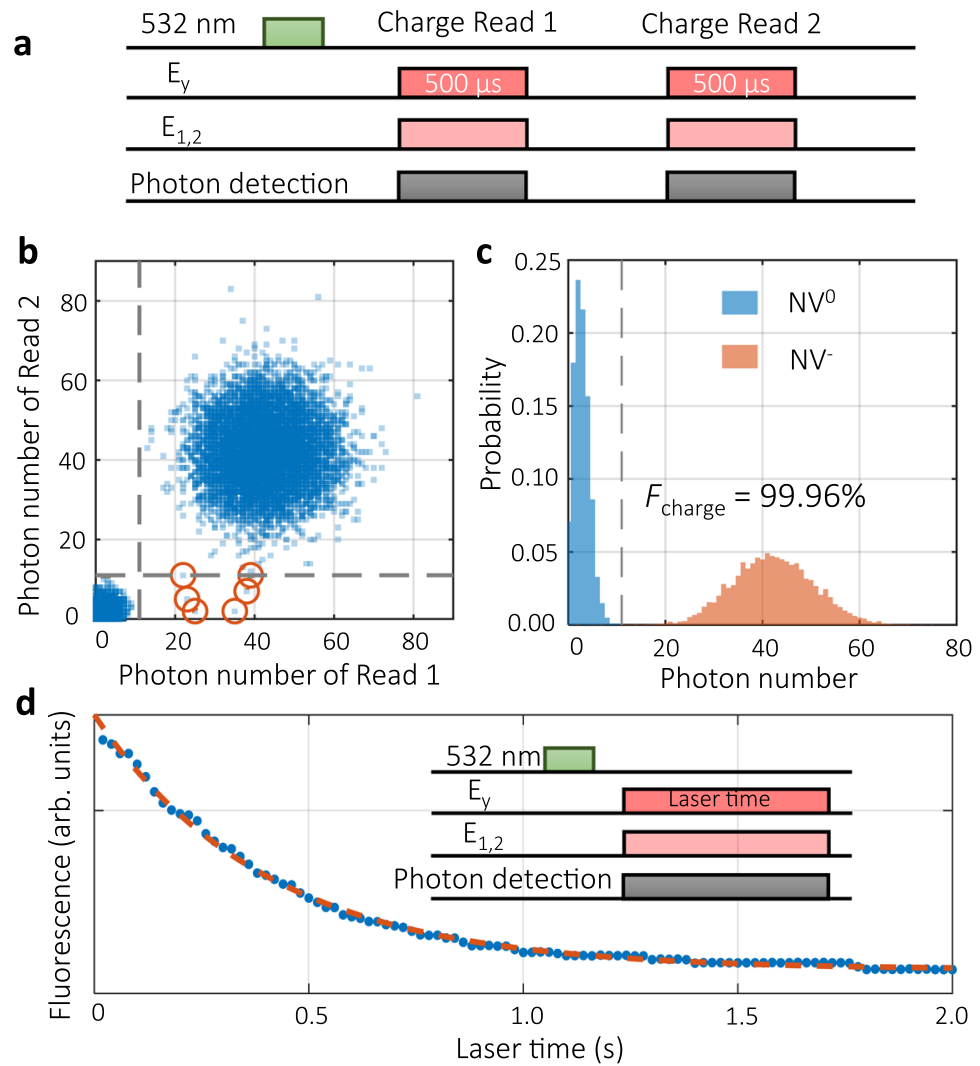
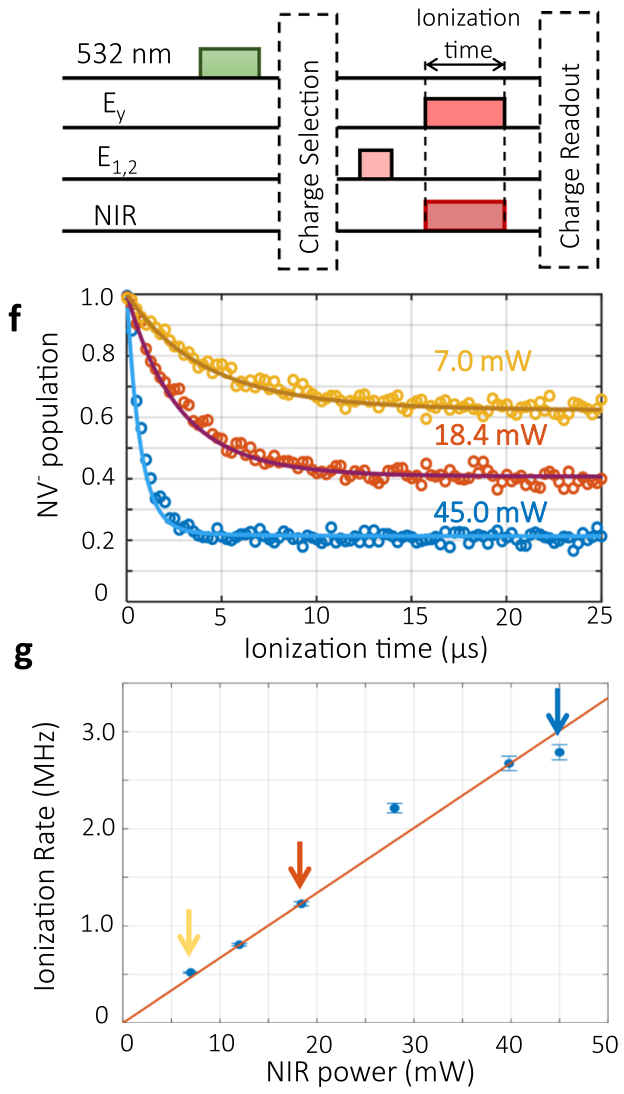

Fig. 2 Non-demolition readout of charge state and ionization rate of NIR light. a Pulse sequence for the charge readout fidelity evaluation. A $3 \mu$ s pulse of $532 \mathrm{~nm}$ laser reset the population of $\mathrm{NV}^{-}$to be $78 \%$, according the results in (b) and (c). Both of the two charge readings use an integration window of $500 \mu \mathrm{s}$. $\mathbf{b}$ The correlation between the two consecutive charge readouts. A total of 10,000 tests are performed. Among them there are 7771 cases of $\left(\mathrm{NV}^{-}, \mathrm{NV}^{-}\right), 6$ cases of $\left(\mathrm{NV}^{-}, \mathrm{NV}^{0}\right), 2223$ cases of $\left(\mathrm{NV}^{0}, \mathrm{NV}^{0}\right)$, and 0 cases of $\left(\mathrm{NV}^{0}, \mathrm{NV}^{-}\right)$. The orange circles mark the cases with anti-correlation. The charge state is judged to be $N V^{0}$ when the collected photon number $\leq 11$. The dashed gray lines mark the threshold (the same for $\mathbf{c}$ ). $\mathbf{c}$ The photon number distribution of $\mathrm{NV}^{0}$ and $\mathrm{NV}^{-}$. The charge readout fidelity $F_{\text {charge }}=99.96 \%$. d The lifetime of the charge state of $\mathrm{NV}^{-}$under $E_{\mathrm{y}}+E_{1,2}(6+5 \mathrm{nW})$ illumination, $400.7 \pm 9.7 \mathrm{~ms}$. e Pulse sequence for measuring the ionization rate under simultaneous illumination of $E_{\mathrm{y}}$ and NIR light. $\mathbf{f}$ The ionization curves of $\mathrm{NV}^{-}$at different powers of $1064 \mathrm{~nm}$. The solid lines are simulations based on different ionization rates. $\mathbf{g}$ The dependence of the NIR ionization rate on its power. The solid line is a linear fit to the data points, with a coefficient of $67.0 \pm 6.7 \mathrm{kHz} / \mathrm{mW}$. The three arrows correspond to the three ionization curves shown in (f).

understand the tiny readout imperfection for $\mathrm{NV}^{-}$state, we measure its lifetime under the continuous optical readout sequence. As shown in Fig. 2d, one observes a lifetime of $400.7 \pm$ $9.7 \mathrm{~ms}$ for $\mathrm{NV}^{-}$state, which causes a charge conversion error of $0.12 \%$ during the charge state readout, comparable to the observed imperfection. The average non-demolition charge readout fidelity is $99.96 \pm 0.02 \%$.

With the non-demolition charge readout, we investigate the ionization by various NIR illumination. We first initialize the charge state to $\mathrm{NV}^{-}$by a $532 \mathrm{~nm}$ laser pulse and measurementbased charge state post-selection. Then a $20 \mu$ s pulse of $E_{1,2}$ initializes the spin to state $|0\rangle$. After the charge and spin initialization, the SCC process is applied, followed by a charge state readout (Fig. 2e). In contrast to the long charge lifetime of 400.7 ms observed in the absence of NIR laser (Fig. 2d), the $\mathrm{NV}^{-}$ population decays fast on the timescale of microseconds after simultaneous illumination of $E_{\mathrm{y}}$ and NIR light (Fig. 2f). However, the $\mathrm{NV}^{-}$population saturation level does not reach at 0 , indicating that in some cases $|0\rangle$ goes through the spin-flip process and gets trapped in $| \pm 1\rangle$, which does not ionize. As the NIR power increases, the $\mathrm{NV}^{-}$population decay faster and saturates at lower levels. To estimate the ionization rate $\Gamma_{\text {ion }}$, we develop an extensive model including a more complicated energy structure as described in Supplementary information. The model uses independently measured quantities and one free parameter $\Gamma_{\text {ion }}$ to fit the data shown in Fig. 2f. The extracted ionization rate is proportional to the NIR laser power (Fig. $2 \mathrm{~g}$ ). This indicates that the NV center is most likely to be ionized from the excited state by absorbing a single $1064 \mathrm{~nm}$ photon. The obtained coefficient of $67.0 \pm 6.7 \mathrm{kHz} / \mathrm{mW}$ is much lower than the $1.2 \pm 0.33 \mathrm{MHz} /$ $\mathrm{mW}$ previously estimated at room temperature ${ }^{24}$, which requires further study in the future.

The highest $\Gamma_{\text {ion }}$ obtained is $2.79 \pm 0.08 \mathrm{MHz}$, only 3.7 times of $\Gamma_{\text {flip }}=0.75 \pm 0.02 \mathrm{MHz}$. One limitation is the output power of current CW NIR laser. The other is the high loss of laser power density on NV center due to transmission reduction and chromatic aberration of the objective. The resulting single-shot fidelity is $89.1 \pm 0.2 \%$ (blue line in Fig. 3c). To improve the conversion efficiency $\left(|0\rangle \rightarrow \mathrm{NV}^{0}\right)$ under current conditions, we consider a correction scheme by utilizing the auxiliary level $m_{\mathrm{S}}=-1$. As shown in Fig. 3a, the leakage population from $|0\rangle$ to the AUX state, is transferred back to $|0\rangle$ state through an $\mathrm{MW}_{\mathrm{AUX}} \pi$ pulse. With this correction, the $|0\rangle$ is converted into $\mathrm{NV}^{0}$ with higher efficiency, while conversion of state $|1\rangle$ is not affected (Fig. 3b). The resulting single-shot fidelity is shown in Fig. 3c. With about $10 \mu$ sCC duration, the average fidelity reaches its maximum of $F_{\text {avg }}=1 / 2\left(F_{|0\rangle}+F_{|1\rangle}\right)=95.4 \pm 0.2 \%$. The corresponding histogram is given in Fig. 3d. We also compare the SCC method with 
a

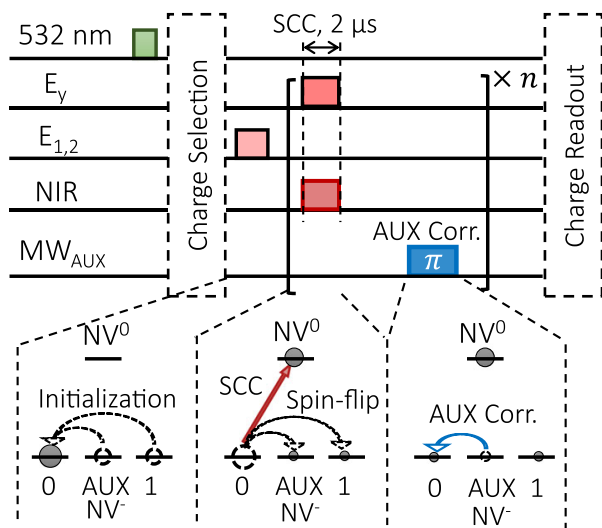

d

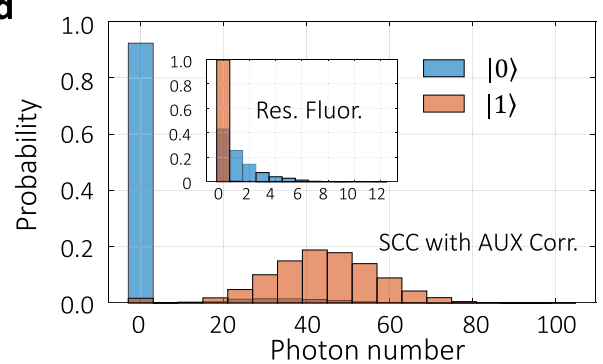

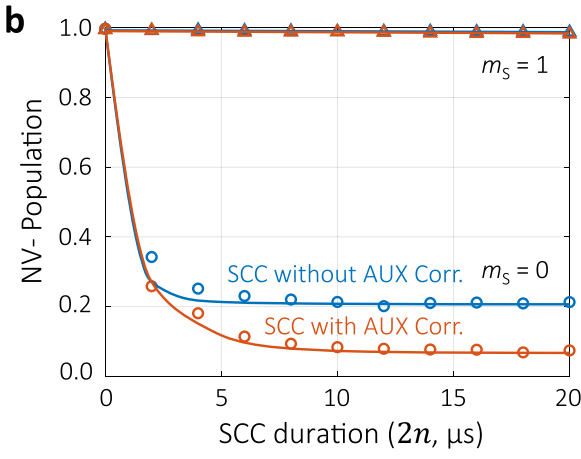

C

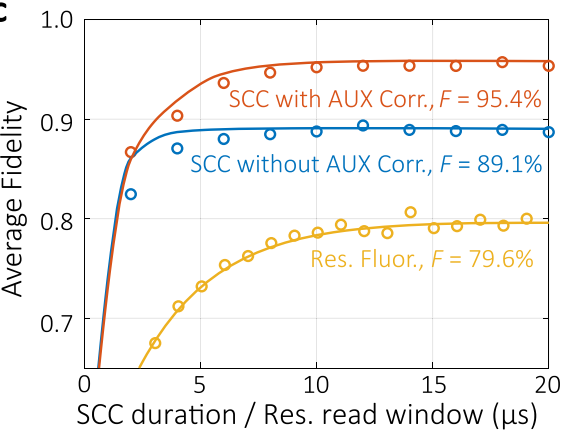

Fig. 3 Single-shot readout of NV electron spin state via SCC. a Pulse sequence and diagram illustrating NV spin and charge dynamics for $|0\rangle$ readout fidelity evaluation. As the spin-flip process traps some populations in $|1\rangle$ and $A U X$ state, an $M W_{A U X} \pi$ pulse rescues the part in $A U X$ state back to $|0\rangle$ so that they can be ionized in the next round. The SCC pulse and AUX correction (AUX Corr.) pulse are repeated for $n$ rounds to get the optimal ionization. The sequence for evaluating |1 readout fidelity only differs in the spin initialization part, which is an $E_{\mathrm{y}}+\mathrm{MW}_{\mathrm{Aux}}$ pulse of $200 \mu \mathrm{s}$. $\mathbf{b} \mathrm{NV}-$ population dependence on SCC duration $(2 \mu \mathrm{s} \times n)$. The solid lines for $|0\rangle$ are simulations. The solid lines for $|1\rangle$ are linear fits to the data. $\mathbf{c}$ Average fidelity dependence on $E_{\mathrm{y}}$ illumination time for different readout methods. In SCC methods $E_{\mathrm{y}}$ illumination time equals the SCC duration, and in resonance fluorescence method it equals the read window. Blue and orange solid lines are the average of the corresponding lines in (b). The yellow line is an exponential fit to the results of the resonance fluorescence (Res. Fluor.) method. $\mathbf{d}$ Photon number distribution of the charge readout with the SCC method. This is obtained from 20,000 measurement repetitions with NV spin initially prepared in the $|0\rangle$ (blue) and $|1\rangle$ (orange). Inset: photon distribution for the resonance fluorescence method.
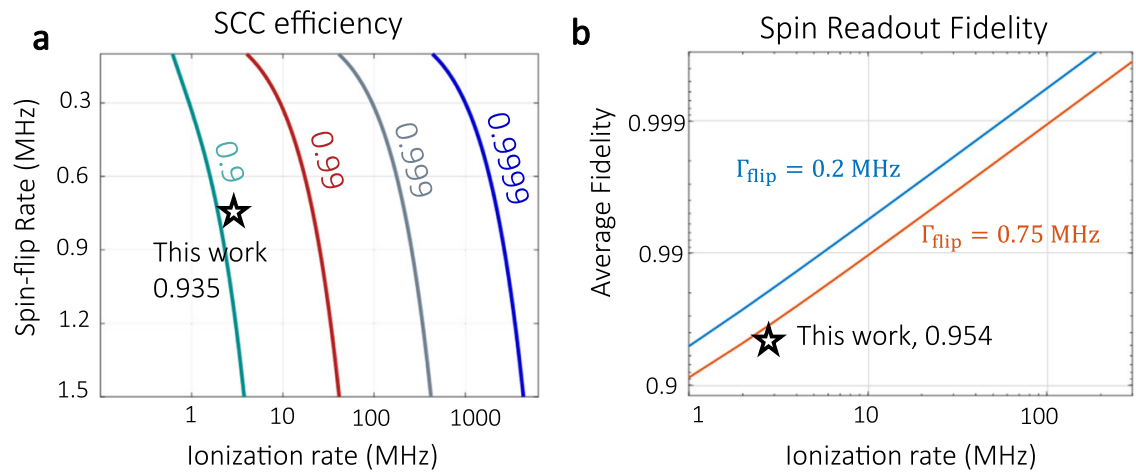

Fig. 4 Exceeding the $\mathbf{9 9 . 9 \%}$ fault-tolerant threshold. a Effects of ionization rate and spin-flip rate on SCC efficiency, which is indicated by the number next to each curve. $\mathbf{b}$ Dependence of overall spin readout fidelity on ionization rate at two different spin-flip rates. The orange line corresponds to spin-flip rate observed in this work. The blue line is a prediction for an NV center with a low spin-flip rate reported in ref. ${ }^{7}$.

the resonance fluorescence method for the single-shot readout. Due to the sizeable spin-flip rate, the optimal average fidelity with resonance fluorescence method is $79.6 \pm 0.8 \%$ (Fig. 3c, d), much lower than previous reports with low-strain NV centers $7,14,15,25$.

\section{Discussion}

The main limiting factor for our single-shot readout fidelity is the SCC efficiency. It depends on both the ionization rate and the spin-flip rate. Figure $4 \mathrm{a}$ shows the simulation results using our model (Supplementary information). The larger ratio $\Gamma_{\text {ion }} / \Gamma_{\text {flip }}$ is, the higher efficiency could be achieved. In practice, $\Gamma_{\text {flip }}$ has a lower bound solely determined by the intrinsic property of NV center. In contrast, $\Gamma_{\text {ion }}$ is convenient to increase by using high power NIR laser and good transmission objective. For a lower $\Gamma_{\text {flip }} \sim 0.2 \mathrm{MHz}^{7}$, a modest NIR power $>1 \mathrm{~W}$ on the diamond could achieve an average single-shot readout fidelity exceeding 99.9\% (Fig. 4b), meeting the requirement for fault-tolerant quantum computing and networks ${ }^{9,26-29}$.

SCC readout is a demolition method for electron spins. Projective readout is still feasible for nuclear spins weakly coupled to the NV center, as their polarization is more robust to the perturbation from optical pumping and ionization ${ }^{30-32}$. The SCC scheme also has the potential for applications on integrated 
quantum devices ${ }^{33-37}$. At present, the photoelectric detection of single NV centers relies on measuring photocurrent from multiple ionizations ${ }^{37}$. The deterministic SCC opens the possibility for achieving optoelectronic single-shot readout of solid spins, potentially utilizing the single-electron transistor as charge reading head ${ }^{38,39}$. Another promising application of single-shot SCC is high-efficiency quantum sensing as discussed in a recent work ${ }^{40}$. Because most of the bio-molecules are rarely affected by the NIR light, the NIR-assisted SCC demonstrated here is helpful to avoid photo-damage on the bio-samples ${ }^{41-44}$.

In summary, we demonstrate a NIR-assisted SCC method for the singe-shot readout of electron spin with fidelity of $95.4 \%$. Different from previous methods which requires careful engineering to improve the emission rate and photon collection efficiency, our method only need an additional NIR beam. By directly controlling the NIR power, the above calculations suggest that the NIR-assisted SCC is an experimentally feasible approach toward spin readout exceeding the fault-tolerant threshold.

We would like to note ${ }^{40}$, which makes use of a similar scheme to achieve single-shot readout with poor optics, using visible, rather than infrared light.

\section{Data availability}

All data needed to evaluate the conclusions in the paper are present in the paper and/or the Supplementary information. Additional data related to this paper may be requested from the authors.

Received: 15 July 2020; Accepted: 7 January 2021;

Published online: 09 March 2021

\section{References}

1. Vamivakas, A. N. et al. Observation of spin-dependent quantum jumps via quantum dot resonance fluorescence. Nature 467, 297-300 (2010).

2. Delteil, A., Gao, W.-B., Fallahi, P., Miguel-Sanchez, J. \& Imamoğlu, A. Observation of quantum jumps of a single quantum dot spin using submicrosecond single-shot optical readout. Phys. Rev. Lett. 112, 116802 (2014).

3. Raha, M. et al. Optical quantum nondemolition measurement of a single rare earth ion qubit. Nat. Commun. 11, 1605 (2020).

4. Kindem, J. M. et al. Control and single-shot readout of an ion embedded in a nanophotonic cavity. Nature 580, 201-204 (2020).

5. Evans, R. E. et al. Photon-mediated interactions between quantum emitters in a diamond nanocavity. Science 362, 662-665 (2018).

6. Bhaskar, M. K. et al. Experimental demonstration of memory-enhanced quantum communication. Nature 580, 60-64 (2020).

7. Robledo, L. et al. High-fidelity projective read-out of a solid-state spin quantum register. Nature 477, 574-578 (2011).

8. Childress, L., Taylor, J. M., Sørensen, A. S. \& Lukin, M. D. Fault-tolerant quantum communication based on solid-state photon emitters. Phys. Rev. Lett. 96, 070504 (2006).

9. Campbell, E. T., Terhal, B. M. \& Vuillot, C. Roads towards fault-tolerant universal quantum computation. Nature 549, 172-179 (2017).

10. Wehner, S., Elkouss, D. \& Hanson, R. Quantum internet: a vision for the road ahead. Science 362, eaam9288 (2018).

11. Awschalom, D. D., Hanson, R., Wrachtrup, J. \& Zhou, B. B. Quantum technologies with optically interfaced solid-state spins. Nat. Photonics 12, 516-527 (2018).

12. Brun, T. A. Quantum error correction. In Oxford Research Encyclopedia of Physics (Oxford Univ. Press, Oxford, 2020).

13. Vahala, K. J. Optical microcavities. Nature 424, 839-846 (2003).

14. Pfaff, W. et al. Unconditional quantum teleportation between distant solidstate quantum bits. Science 345, 532-535 (2014).

15. Humphreys, P. C. et al. Deterministic delivery of remote entanglement on a quantum network. Nature 558, 268-273 (2018).

16. Abobeih, M. H. et al. Atomic-scale imaging of a 27-nuclear-spin cluster using a quantum sensor. Nature 576, 411-415 (2019).

17. Lončar, M. \& Faraon, A. Quantum photonic networks in diamond. MRS Bull. 38, 144-148 (2013).

18. Shields, B. J., Unterreithmeier, Q. P., de Leon, N. P., Park, H. \& Lukin, M. D. Efficient readout of a single spin state in diamond via spin-to-charge conversion. Phys. Rev. Lett. 114, 136402 (2015).
19. Hopper, D. A., Grote, R. R., Exarhos, A. L. \& Bassett, L. C. Near-infraredassisted charge control and spin readout of the nitrogen-vacancy center in diamond. Phys. Rev. B 94, 241201 (2016).

20. Jaskula, J.-C. et al. Improved quantum sensing with a single solid-state spin via spin-to-charge conversion. Phys. Rev. Appl. 11, 064003 (2019).

21. Hopper, D. A., Grote, R. R., Parks, S. M. \& Bassett, L. C. Amplified sensitivity of nitrogen-vacancy spins in nanodiamonds using all-optical charge readout. ACS Nano 12, 4678-4686 (2018).

22. Jayakumar, H., Dhomkar, S., Henshaw, J. \& Meriles, C. A. Spin readout via spin-to-charge conversion in bulk diamond nitrogen-vacancy ensembles. Appl. Phys. Lett. 113, 122404 (2018).

23. Hopper, D. A., Lauigan, J. D., Huang, T.-Y. \& Bassett, L. C. Real-time charge initialization of diamond nitrogen-vacancy centers for enhanced spin readout. Phys. Rev. Appl. 13, 24016 (2020).

24. Meirzada, I., Hovav, Y., Wolf, S. A. \& Bar-Gill, N. Negative charge enhancement of near-surface nitrogen vacancy centers by multicolor excitation. Phys. Rev. B 98, 245411 (2018)

25. Bernien, H. et al. Heralded entanglement between solid-state qubits separated by three metres. Nature 497, 86-90 (2013).

26. Muralidharan, S., Kim, J., Lütkenhaus, N., Lukin, M. D. \& Jiang, L. Ultrafast and fault-tolerant quantum communication across long distance. Phys. Rev. Lett. 112, 250501 (2014).

27. Waldherr, G. et al. Quantum error correction in a solid-state hybrid spin register. Nature 506, 204-207 (2014).

28. Rong, X. et al. Experimental fault-tolerant universal quantum gates with solidstate spins under ambient conditions. Nat. Commun. 6, 8748 (2015)

29. Yang, S. et al. High-fidelity transfer and storage of photon states in a single nuclear spin. Nat. Photonics 10, 507-511 (2016).

30. Jiang, L. et al. Repetitive readout of a single electronic spin via quantum logic with nuclear spin ancillae. Science 326, 267-272 (2009).

31. Neumann, P. et al. Single-shot readout of a single nuclear spin. Science 329, 542-544 (2010).

32. Maurer, P. C. et al. Room-temperature quantum bit memory exceeding one second. Science 336, 1283-1286 (2012).

33. Brenneis, A. et al. Ultrafast electronic readout of diamond nitrogen-vacancy centres coupled to graphene. Nat. Nanotechnol. 10, 135-139 (2015).

34. Bourgeois, E. et al. Photoelectric detection of electron spin resonance of nitrogen-vacancy centres in diamond. Nat. Commun. 6, 8577 (2015).

35. Gulka, M. et al. Pulsed photoelectric coherent manipulation and detection of NV centre spins in diamond. Phys. Rev. Appl. 7, 044032 (2016).

36. Hrubesch, F. M., Braunbeck, G., Stutzmann, M., Reinhard, F. \& Brandt, M. S Efficient electrical spin readout of NV- centers in diamond. Phys. Rev. Lett. 118, 037601 (2017).

37. Siyushev, P. et al. Photoelectrical imaging and coherent spin-state readout of single nitrogen-vacancy centers in diamond. Science 363, 728-731 (2019).

38. Yin, C. et al. Optical addressing of an individual erbium ion in silicon. Nature 497, 91-94 (2013)

39. Zhang, Q. et al. Single rare-earth ions as atomic-scale probes in ultrascaled transistors. Nano Lett. 19, 5025-5030 (2019).

40. Irber, D. M. et al. Robust all-optical single-shot readout of NV centers in diamond. Nat. Commun. 12, 532 (2021)

41. Shi, F. et al. Single-protein spin resonance spectroscopy under ambient conditions. Science 347, 1135-1138 (2015)

42. Shi, F. et al. Single-DNA electron spin resonance spectroscopy in aqueous solutions. Nat. Methods 15, 697-699 (2018).

43. Cheng, Y. Single-particle cryo-EM-how did it get here and where will it go. Science 361, 876-880 (2018)

44. Yang, Y. et al. A reactive, rigid Gd III labeling tag for In-Cell EPR distance measurements in proteins. Angew. Chem. Int. Ed. 56, 2914-2918 (2017).

45. Togan, E. et al. Quantum entanglement between an optical photon and a solid-state spin qubit. Nature 466, 730-734 (2010).

\section{Acknowledgements}

The authors are grateful to Sven Rogge, Milos Nesladek, Friedemann Reinhard, and Guanglei Cheng for helpful discussions. This work is supported by the National Key R\&D Program of China (Grant Nos. 2018YFA0306600, 2017YFA0305000, 2016YFA0502400, 2019YFA0709300), the NNSFC (Grants Nos. 11775209, 81788101, 11761131011, 11722544, 91636217, 31971156), the CAS (Grants Nos. GJJSTD20200001, QYZDY-SSW-SLH004), Anhui Initiative in Quantum Information Technologies (Grant No. AHY050000), the Fundamental Research Funds for the Central Universities.

\section{Author contributions}

J.D. supervised the project; Q.Z. and Y.W. conceived the ideas and supervised the experiments; W.J. and Y.G. built the setup; M.W. prepared the sample; Y.G., W.J. and J.Y. performed the experiments; Y.G., Q.Z. and W.J. conducted the simulations; Q.Z., Y.W., Y.G. and W.J. wrote the manuscript. All authors along analyzed the data and revised the manuscript. 


\section{Competing interests}

The authors declare no competing interests.

\section{Additional information}

Supplementary information The online version contains supplementary material available at https://doi.org/10.1038/s41467-021-21781-5.

Correspondence and requests for materials should be addressed to Y.W. or J.D.

Peer review information Nature Communications thanks the anonymous reviewer(s) for their contribution to the peer review of this work.

Reprints and permission information is available at http://www.nature.com/reprints

Publisher's note Springer Nature remains neutral with regard to jurisdictional claims in published maps and institutional affiliations. (c) Open Access This article is licensed under a Creative Commons Attribution 4.0 International License, which permits use, sharing, adaptation, distribution and reproduction in any medium or format, as long as you give appropriate credit to the original author(s) and the source, provide a link to the Creative Commons license, and indicate if changes were made. The images or other third party material in this article are included in the article's Creative Commons license, unless indicated otherwise in a credit line to the material. If material is not included in the article's Creative Commons license and your intended use is not permitted by statutory regulation or exceeds the permitted use, you will need to obtain permission directly from the copyright holder. To view a copy of this license, visit http://creativecommons.org/ licenses/by/4.0/.

(c) The Author(s) 2021 